\title{
Design, modeling and identification of an ultrasonic composite transducer for target impedance independent short pulse generation
}

\author{
Fabian Bause $^{1}$, Jens Rautenberg ${ }^{1}$, Bernd Henning ${ }^{1}$ \\ 1 University of Paderborn, Measurement Engineering Group, Warburger Str. 100, 33098 Paderborn, \\ Corresponding author's e-mail address: bause@emt.uni-paderborn.de
}

\begin{abstract}
The design and modeling of an ultrasonic 1-3-composite transducer is presented. We describe a successive identification method of the complete transducer, consisting of a front protection layer, a backing and an electrical matching network. Furthermore we discuss the influence of the internal impedance of the electrical source driving the transducer. The aim of this contribution is to provide a complete identified transducer model, to characterize influences of the assembling process to the electro-mechanical behavior and finally to design a high bandwidth composite transducer.
\end{abstract}

Key words: Identification, 1-3 composite, modeling, high bandwidth

\section{Introduction}

In the field of ultrasonic measurement short ultrasonic pulses are an essential component for many applications. On the one hand short pulses give a better spatial resolution regarding ultrasonic imaging in medicine or nondestructive testing. On the other hand they are of great value when one intends to identify different propagation paths e.g. in acoustic waveguides. As shown in [1], the identification of multiple propagation paths in acoustic waveguides is well suited for material identification purposes, which will be the aiming application in this contribution. Furthermore it is worth to note that the considered target materials are polymers.

\section{Transducer design}

With respect to the considered application we need to formulate some basic requirements:

- Impedance matching between transducer and target material for bandwidth improvement

- Uniform plane displacements with preferably very low tangential stress components for homogeneous and defined excitation of the waveguide structure

- Modeling should be possible in only one dimension
These requirements can be met by using a 1-3 piezocomposite as active element instead of a common bulk piezoceramic disc.

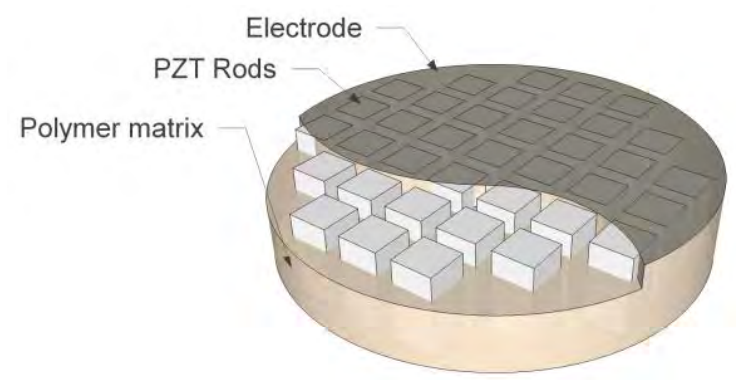

Fig. 1. Schematic of a 1-3 composite disc.

A piezocomposite consists of a combination of piezoceramic elements and a passive polymer. Thus, the effective acoustic impedance of such a composite is lower than the original impedance of the active material. Furthermore, the geometrical structure of a 1-3 composite (see Figure 1) suppresses parasitical radial oscillations which results in a homogeneous oscillation in thickness mode direction. For modeling purposes we choose a disc shaped active element. A protection layer between target material and composite is needed to safeguard against thermal, chemical and mechanical influences. Furthermore we want this layer to act as mass contact to prevent nonsymmetrical influences of wrap-around electrodes. Therefore we use a stainless steel protection layer which is adhered to the 1-3 composite using an acrylate glue (Loctite 638). The adhesive it-self is not conducting, hence 
the thickness of this layer has to be as small as possible to achieve an electrical resistive contact between both bonded parts. The backing material consist of a mixture of a 2component polyurethane and fused tungsten carbides which ensures good acoustic coupling, high damping properties and good mechanical stability.

\section{Transducer modeling and identification}

In the following we describe the modeling and identification process of the proposed transducer. Beginning with the active element it-self, we describe the construction, modeling and identification stepwise up to the complete transducer.

\section{Composite disc}

We model the 1-3 composite disc based on the well-known mason model (3-port). Hence, we can simply add several layers, such as adhesive layers or a protection layer, by describing them as transmission lines (2-ports). For identification purposes of the active element we need to work with effective parameters, as the mason model relies on homogeneous structures. Furthermore, it is worth noting, that most parameters cannot be measured directly and therefore have to be estimated based on ANSI/IEEE 176 [2] and DIN IEC-60483 [3]. Measureable parameters of the active element are:

- Thickness of composite disc: $t$

- Diameter of composite disc : $D$

- Mass of composite disc: $m$

- $\quad$ "Free" capacity at $1 \mathrm{kHz}: C_{0}^{T}$

- Series resonance (thickness mode): $f_{s}$

- Antiresonance (thickness mode): $f_{a}$

- Electrical impedance at $f_{s}: Z_{\min }$

- Series resonance (radial mode): $f_{r, 1}$

- Antiresonance (radial mode): $f_{a, 1}$

- Electrical impedance at $f_{r, 1}: Z_{\min , 1}$

- Series impedance ( $1^{\text {st }}$ radial harmonic): $f_{r, 2}$

First we need to estimate a value for the coupling factor of the thickness mode. Following ANSI/IEEE 176 we get:
$k_{T}^{2} \approx \frac{\pi}{2} \frac{f_{s}^{\prime}}{f_{a}^{\prime}} \tan \left(\frac{\pi}{2} \frac{\Delta f}{f_{a}^{\prime}}\right) \quad \Delta f=f_{a}^{\prime}-f_{s}^{\prime}$.

Here the primed quantities denote resonance and antiresonance frequency at zero phase. If these quantities are unknown one can at least estimate their difference [4]:

$$
\Delta f \approx \frac{f_{a}-f_{s}}{\sqrt{1+16\left(\pi f_{s} C_{0}^{T} Z_{\text {min }}\right)^{2}}} .
$$

For further calculations we need to note that in fact our real composite disc is a compound of active element and copper-tin (CuSn) electrodes. For decomposition purposes we assume the electrode material's density and wave velocity to be $\rho_{e}=8500 \mathrm{~g} / \mathrm{l}$ and $c_{e}=5227 \mathrm{~m} / \mathrm{s}$ respectively. The effective density of the composite-electrode-compound is defined as

$\rho_{e f f}=\frac{m}{t \pi D^{2} / 4}$.

Using ANSI/IEEE 176 [2] we can estimate the electrode's thickness $t_{e}$ :

$t_{e} \approx \frac{t \rho_{e f f}}{2 \rho_{e}} \frac{\Delta f / f_{a}-4 k_{T}^{2} / \pi^{2}}{1+\Delta f / f_{a}-4 k_{T}^{2} / \pi^{2}}$

and therefore we can compute the composite material's density:

$\rho=\frac{t \rho_{e f f}-2 t_{e} \rho_{e}}{t-2 t_{e}}$.

The effective sound velocity in thickness direction of the composite material can be calculated using resonance condition:

$c_{T}^{D}=2 \tilde{t} f_{a} \quad \tilde{t}=t+2 t_{e}\left(c_{T}^{D} / c_{e}+1\right)$

with $\tilde{t}$ being an effective thickness taking electrodes with specified sound velocity and dimension into account. Hence we get:

$c_{T}^{D}=\frac{2 f_{a}\left(t-2 t_{e}\right)}{1-4 t_{e} f_{a} / c_{e}}$.

For calculating the "free" permittivity (constant stress) we need to consider, that the electric field within the electrodes is zero.

$\varepsilon_{33}^{T}=\frac{C_{0}^{T}\left(t-2 t_{e}\right)}{\pi D^{2} / 4}$

For modeling purposes we need to estimate a value for the "clamped" permittivity (constant 
strain). From ANSI/IEEE 176 and DIN IEC60483 we get:

$\varepsilon_{33}^{S} \approx\left(1-k_{T}^{2}\right)\left(1-k_{P}^{2}\right) \varepsilon_{33}^{T}$.

The coupling factor of the planar oscillation $k_{P}$ can be estimated using poission's ratio $v$ as well as the resonances and antiresonances of the radial mode [2]:

$k_{P}^{2} \approx \frac{\tilde{J}_{1}\left(\xi_{1}\right)+v-1}{\tilde{J}_{1}\left(\xi_{1}\right)-2} \quad \tilde{J}_{1}\left(\xi_{1}\right)=\frac{\xi_{1} J_{0}\left(\xi_{1}\right)}{J_{1}\left(\xi_{1}\right)}$,

with $J_{n}(x)$ being ordinary bessel functions of order $n$. The functions argument reads

$\xi_{1}=\eta_{1}\left(1+\frac{\Delta f_{1}}{f_{r, 1}}\right)$,

with $\Delta f_{1}$ being calculated analogue to eq. (2). The poission's ratio and parameter $\eta_{1}$ can be estimated using table 12 in ANSI/IEEE 176 as function of $f_{r, 2} / f_{r, 1}$. The composite's "clamped" capacity can now be expressed as

$C_{0}^{S}=\frac{\varepsilon_{33}^{S} \pi D^{2}}{4\left(t-2 t_{e}\right)}$.

For modeling purposes the mechanical quality factor is needed as well, which can be estimated as follows [4]:

$Q_{m} \approx\left(2 \pi f_{r} Z_{\min } C_{0}^{S} \frac{\Delta f^{2}}{f_{a}^{2}}\right)^{-1}$.

Using these quantities as defined above one is able to compute the piezoelectric constant $h_{33}$, the acoustic radiation impedance $Z_{m}$ and complex wavenumber $\gamma$, which are necessary for describing a complete impedance matrix used for the proposed 3-port mason model $[5,1]$ :

$$
\begin{aligned}
& h_{33}=c_{T}^{D} \sqrt{\frac{k_{T}^{2} \rho}{\varepsilon_{33}^{S}}}, \\
& Z_{m} \approx \rho c_{T}^{D}\left(\pi D^{2} / 4\right)\left(1+\frac{j}{2 Q_{m}}\right), \\
& \gamma=j k \approx \frac{\omega}{c_{T}^{D}}\left(\frac{1}{2 Q_{m}}+j\right)\left(1+\frac{1}{Q_{m}^{2}}\right)^{-1 / 2} .
\end{aligned}
$$

The electric input impedance can now be calculated and compared to the measured data. Because most of the quantities are estimates, it is admissible to optimize certain parameters to achieve a better match between measurement and simulation. In particular we propose to optimize the composite material's density and sound velocity as well as the "clamped" permittivity and mechanical quality factor.

As can be seen in Fig. 2, the simulation using estimated parameters is very close to our measurement. After optimizing the four proposed parameters, the match between simulation and measurement is even better. The optimized parameters differ only slightly from their first estimates. The variation of density and speed of sound is below $+0.1 \%$. The optimized "clamped" permittivity is $7.9 \%$ smaller than its first estimate and the optimized quality factor is $3.36 \%$ larger than its estimate.

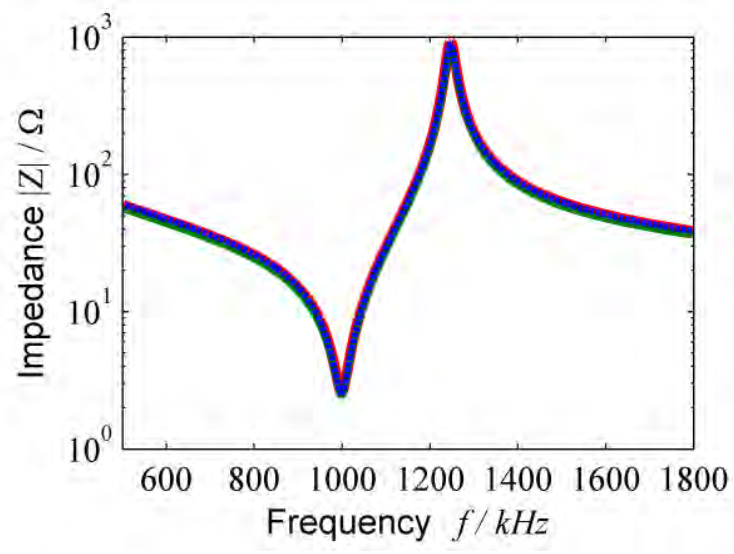

Fig. 2. Electrical input impedance of a composite disc. Depicted are a measurement (red), a simulation using estimated parameters (green) and a simulation using optimized parameters (dashed blue).

Protection layer and adhesive

A protection layer made of stainless steel between target material and composite is adhered to the composite's face to safeguard against thermal, chemical and mechanical influences. The steel foil's thickness is $77 \mu \mathrm{m}$. It is worth to note that the thickness of the steel foil and the thickness of the adhesive layer will affect the thickness mode resonances. Hence, the composite's thickness and therefore the location of the resonance of the thickness mode has to be chosen such, that the resulting compound of composite, adhesive layer and steel foil shows the desired thickness mode characteristics. We use deep drawing technique to recast the metal foil into a pot-like structure. In its center we adhere the composite disc using Loctite 638. The adhesive layer's thickness is about $20-25 \mu \mathrm{m}$.

Using the parameterized mason model and two 2-port models representing the steel and adhesive layer it should be possible to simulate the electrical input impedance properly. But as can be seen in Fig. 3, the adhering process 
massively affects the composite disc's properties. As we apply chemical (adhesive and activator), thermal (tempering at $353 \mathrm{~K}$ ) and mechanical (pressing) stresses to the compound all at once to achieve a thin electrically conduction and mechanically stable adhesive layer, the reasons for these changes are not yet understood. Nevertheless, it is possible to estimate the resulting properties of the composite using the proposed optimization process once again. We assume constant material properties for the adhesive and steel layer, which is of course a bottleneck of this procedure, and optimize the quality factor, the coupling factor of the thickness mode and the "clamped" permittivity. The result of this optimization is depicted in Fig. 3 (blue curve).

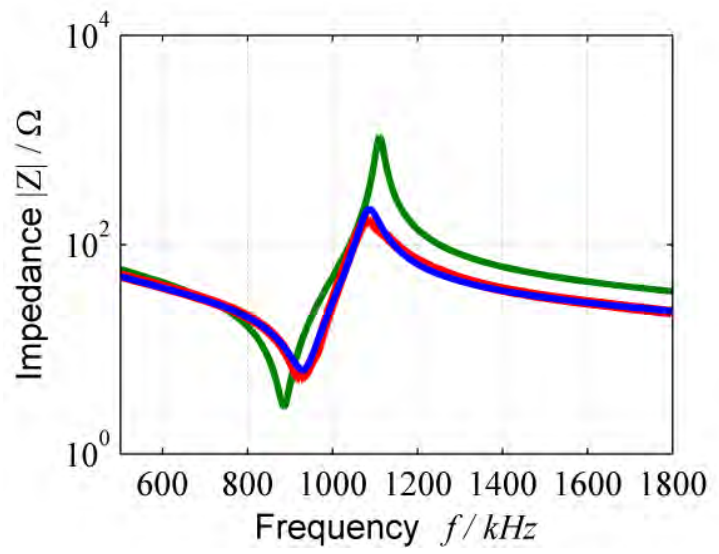

Fig. 3. Electrical input impedance of the compound of composite disc, adhesive layer and metal foil. Depicted are a measurement (red), simulation without adjusted parameters (green) and simulation with optimized parameters (blue).

\section{Backing}

To decrease the quality factor and therefore widening the transducers bandwidth we employ a backing material consisting of a 2-component polyurethane (PUR) and fused tungsten carbides, in the following tungsten carbide for short. Knowing the acoustical properties of PUR and tungsten carbide [8, p. 14-46], it is possible to calculate the properties of the resulting random 0-3 composite $[6,7]$. The acoustic properties and density of PUR have been measured using time-of-flight (TOF) measurements for longitudinal wave speed calculation, shore-hardness measurements to estimate shear wave speed [9] and gravimetric measurements for density calculation. Using the 0-3 composite model as proposed by Devaney and Levine [7] we calculate the effective acoustic impedance as function of mass fraction of tungsten carbide (see Fig. 4).

Searching for a good backing impedance we consider the transmit transfer function (TTF) and its inverse Fourier transform which is the impulse response of our electro-mechanical system. More precisely, we investigate the simulated mechanical vibration of the transducer given an electrical pulse (Gaussian modulated sine with $1.1 \mathrm{MHz}$ center frequency and $120 \%$ bandwidth). Given a range of target impedances of 1.5 MRayl to 4 MRayl we focus on the transient oscillations while varying the backing impedance. We found, that a backing impedance of approx. $6.5 \mathrm{MRayl}$ results in a good compromise between a short pulse and smooth transient oscillations (see Fig. 5).

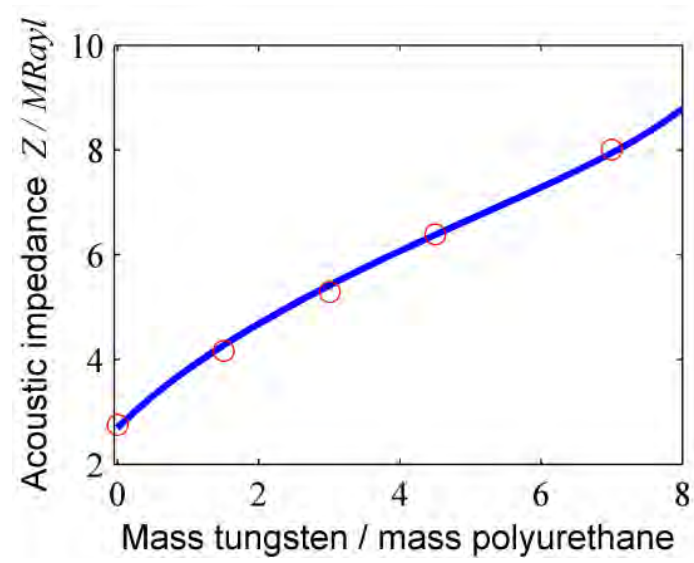

Fig. 4. Effective acoustic impedance of 0-3 composite consisting of PUR and tungsten carbide. Blue: Model as proposed by Devaney and Levine. Red: Calculated acoustic impedance using TOF measurements and modeled effective density.

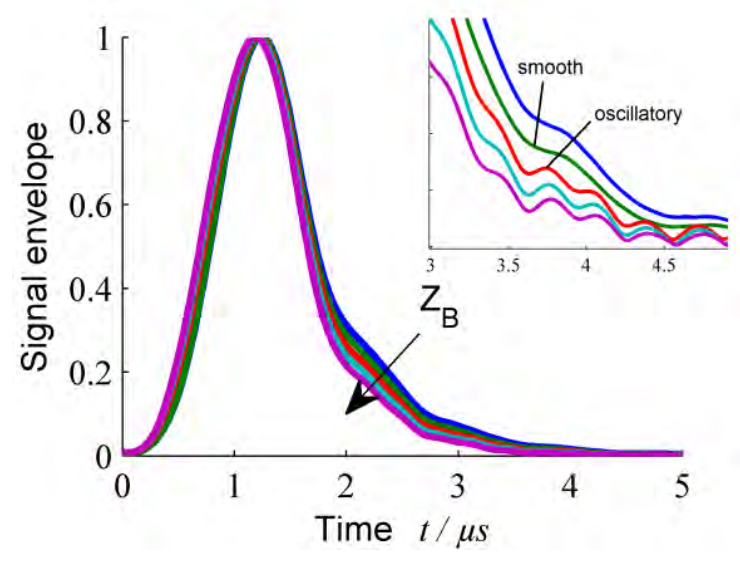

Fig. 5. Normalized envelope of simulated mechanical vibration given a target impedance of 3 MRayl and varying backing impedances $\left(Z_{\mathrm{B}}\right)$ from 6 MRayl to 8 MRayl. Step width is 0.5 MRayl. Upper right: Zoom into $3 \mu$ s to $4.8 \mu$ s.

\section{Electrical matching network}

We part the transducer's electrical network into three segments. Internal impedance of electrical source, electric cable and electrical matching network. All segments can easily be added as (electrical) 2-ports into our existing model, presupposed they can be identified well. Considering the internal resistance of our electrical source we need to identify the output 
impedance of our amplifier, which consists of a full bridge of two operational amplifiers (LTI 1210) and an output transformer. Thus we need to consider the transformed output resistance of the operational amplifiers and the inductivity of the output transformer.

As electric cable, connecting amplifier and transducer we use a coaxial cable of $1.5 \mathrm{~m}$ (Bedea RG174). Its properties for transmission line modeling (resistance and capacity per unit length) are given within its datasheet.

Calculating the TTF as seen from an ideal electrical source, one can observe that the ohmic internal impedance of the electrical source shifts the maximum of the TTF to higher frequencies. Furthermore, the resistance effects the bandwidth and thus the damping behavior of the complete transducer. Thus, we design an electrical matching network consisting of a resistance and an additional inductivity, which acts in combination with the capacitance of the transducer as oscillating circuit and thus can increase the transducers bandwidth if chosen properly. We search for a combination of resistance and inductivity, to reach a smooth and broadband TTF. Fig. 6 illustrates the TTF without matching network and with a matching network consisting of a $27 \Omega$ resistor and an inductivity of $2.45 \mu \mathrm{H}$. The effect of the proposed electrical network on the acoustic signal can be seen in Fig. 7. For both simulations the internal impedance of the electrical source has been considered. The phase shift is due to the inductivity of the matching network, whereas the reduction in amplitude is mainly due the resistor.

\section{Validation and conclusions}

For validation purposes of the complete model we consider once more the electro-mechanical domain (electrical input impedance) and moreover we take acoustic measurements using a hydrophone (RP $15 \mathrm{~s}$, RP acoustics).

As can be seen in Fig. 8 the modeling of the electrical matching network works fine. Furthermore, we note some discrepancies between measurement and simulation concerning the damping of the thickness mode oscillation, which is simulated slightly too high.

For acoustic measurements the transducer is placed on the surface of a water bath. At a distance of approx. $100 \mathrm{~mm}$ (far field conditions) we place a hydrophone recording the transmitted signals. Please note, that due to the actual architecture of the amplifier no inductivity for electrical matching could be used. Fig. 9 illustrates a comparison between simulation and measurement. For comparison purposes the measured and simulated signal have been normalized and shifted in time.

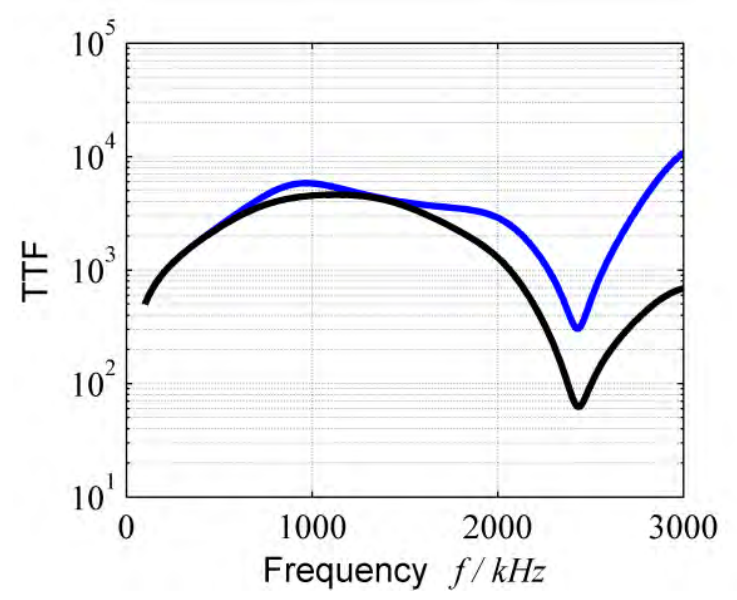

Fig. 6. Simulated transmit transfer function of transducer without electrical matching network (blue) and with electrical matching network (black). Assumed for simulation are a 3 MRayl target impedance and a 6.5 MRayl backing impedance.

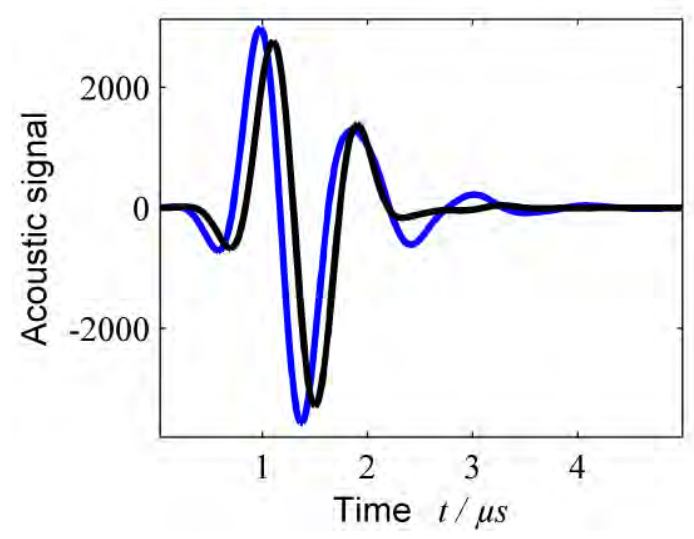

Fig. 7. Simulated acoustic signal without electrical matching network (blue) and with electrical matching network (black). Target as well as backing impedances and colors consistent to Fig. 6.

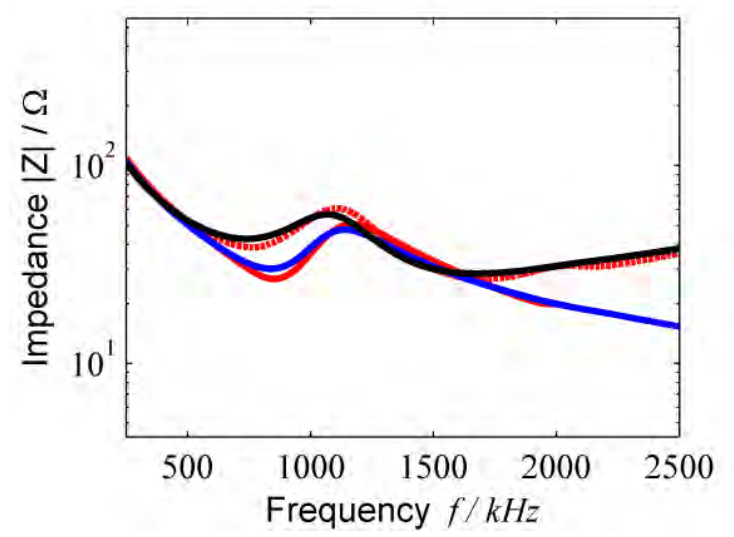

Fig. 8. Electrical input impedance of complete transducer with (black and dashed red curve) and without electrical matching network (blue and solid red curve). The red curves delineate measurements. 
It can be seen, that the general pulse shape is very short due to the high bandwidth behavior of the transducer and furthermore can be simulated quite well. Discrepancies are only found within the pulse's symmetry.

The slight discrepancies still found between measurements and simulations may be caused by the impulse response of the hydrophone and the lack of information on the adhering process of the front protection layer. As outlined above, the combination of thermal, chemical and mechanical influences on the composite while adhering and tempering is very complex. The actual method to optimize the parameters of the composite disc once more after adhering does not result in an optimal match (Fig. 3). One reason may be the cross-sensitivity to uncertain properties of the adhering and protection layer, which affects the optimization process.

Hence, in further studies, we want to focus on this adhering process to reduce the influences and make them describable with respect to the composite's changes. Moreover, the influence of the impulse response of the hydrophone may be compensated using deconvolution technique [10].

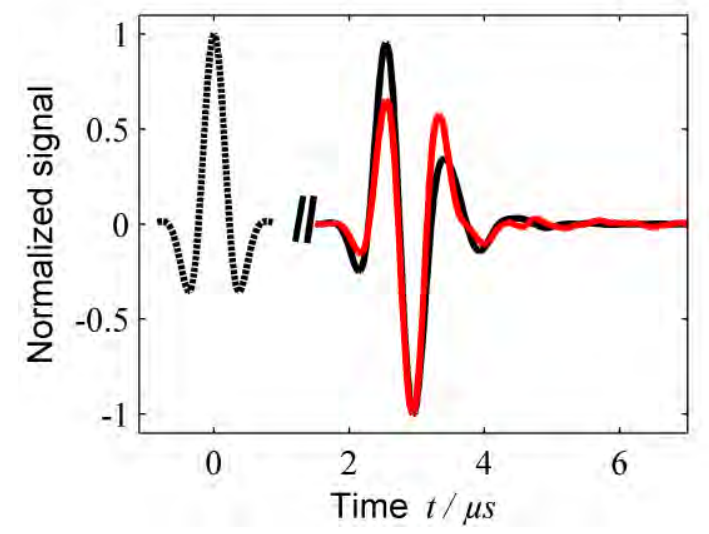

Fig. 9. Simulated (black) and measured (red) signal of the proposed transducer. Target impedance: 1.48 MRayl (water). Dashed black: sending signal.

\section{Acknowledgements}

We would like to thank Eugen Djakow, Forming and Machining Technology, University of Paderborn, for his advice and support regarding deep drawing technology. Moreover, we want to thank the German Research Foundation (DFG) for financial support of the research project $\mathrm{HE}$ 2897/3-1 (determination of acoustical material properties).

\section{References}

[1] J. Rautenberg, Ein wellenleiterbasiertes Verfahren zur Bestimmung von Materialdaten für die realitätsnahe Simulation von Schallausbreitungsphänomenen am Beispiel stark absorbierender Kunststoffe, Dissertation, Universität Paderborn (2012)

[2] American National Standard Institute, IEEE Standard on Piezoelectricity, ANSI/IEEE Std. 176, The Institute of Electrical and Electronics Engineers, Inc., New York 1988

[3] International Electrotechnical Commission, Leitfaden zur Bestimmung der dynamischen Eigenschaften von piezoelektrischer Keramik mit hohem elektromechanischem Koppelfaktor, DIN IEC-60483, Genf 1976

[4] T. R. Gururaja, W. A. Schulze, L. E. Cross et al, Piezoelectric Composite Materials for Ultrasonic Transducer Applications. Part I: Resonant Modes of Vibration of PZT Rod-Polymer Composites, IEEE Transactions on Sonics and Ultrasonics, Vol. SU-32, No. 4, July 1985

[5] R. Lerch, G. Sessler, D. Wolf, Technische Akustik: Grundlagen und Anwendungen, Springer Verlag, ISBN: 3540234306, 2009

[6] H. Wang, T. Ritter, W. Cao, K. Shung, High Frequency Properties of Passive Materials for Ultrasonic Transducers, Trans. UFFC, Vol. 48, No. 4, pp. 78-84, January 2001

[7] A. J. Devaney and H. Levine, Effective elastic parameters of random composites, Appl. Phys. Lett. Vol. 37, pp. 377-379, 1980

[8] W. M. Haynes (Ed.), CRC Handbook of Chemistry and Physics, 93th edition, Taylor \& Francis, July 2012

[9] H. J. Qi, K. Joyce, M. C. Boyce: Durometer hardness and the stress-strain behavior of elastomeric materials, Rubber Chemistry and Technology, Vol. 76, No. 2, pp. 419-435, 2003

[10] V. Wilkens, C. Koch: Improvement of hydrophone measurements on diagnostic ultrasound machines using broadband complex-valued calibration data, Journal of Physics: Conference Series 1, pp. 50-55, 2004 